\title{
A gene-based SNP resource and linkage map for the copepod Tigriopus californicus
}

\author{
Brad R Foley ${ }^{1}$, Colin G Rose ${ }^{1,2}$, Daniel E Rundle ${ }^{1,3}$, Wai Leong ${ }^{1}$, Gary W Moy ${ }^{4}$, Ronald S Burton ${ }^{4}$ and \\ Suzanne Edmands ${ }^{1 *}$
}

\begin{abstract}
Background: As yet, few genomic resources have been developed in crustaceans. This lack is particularly evident in Copepoda, given the extraordinary numerical abundance, and taxonomic and ecological diversity of this group. Tigriopus californicus is ideally suited to serve as a genetic model copepod and has been the subject of extensive work in environmental stress and reproductive isolation. Accordingly, we set out to develop a broadly-useful panel of genetic markers and to construct a linkage map dense enough for quantitative trait locus detection in an interval mapping framework for T. californicus-a first for copepods.

Results: One hundred and ninety Single Nucleotide Polymorphisms (SNPs) were used to genotype our mapping population of $250 \mathrm{~F}_{2}$ larvae. We were able to construct a linkage map with an average intermarker distance of 1.8 $\mathrm{CM}$, and a maximum intermarker distance of $10.3 \mathrm{cM}$. All markers were assembled into linkage groups, and the 12 linkage groups corresponded to the 12 known chromosomes of $T$. californicus. We estimate a total genome size of $401.0 \mathrm{cM}$, and a total coverage of $73.7 \%$. Seventy five percent of the mapped markers were detected in 9 additional populations of $T$. californicus. Of available model arthropod genomes, we were able to show more colocalized pairs of homologues between T. californicus and the honeybee Apis mellifera, than expected by chance, suggesting preserved macrosynteny between Hymenoptera and Copepoda.

Conclusions: Our study provides an abundance of linked markers spanning all chromosomes. Many of these markers are also found in multiple populations of $T$. californicus, and in two other species in the genus. The genomic resource we have developed will enable mapping throughout the geographical range of this species and in closely related species. This linkage map will facilitate genome sequencing, mapping and assembly in an ecologically and taxonomically interesting group for which genomic resources are currently under development.
\end{abstract}

\section{Background}

Copepods are among the most abundant metazoans on the planet and include more than 11,500 described species [1]. They are ecologically important, spanning a broad range of marine, freshwater and moist terrestrial habitats and serving as major players in global energy transfer, including the largest portion of carbon turnover in the oceanic food web [2]. They are also of economic interest, serving as both prey and parasites of valuable fishery species. Despite their ecological and economic importance, there are few genetic resources developed

\footnotetext{
* Correspondence: sedmands@usc.edu

'Department of Biological Sciences, University of Southern California, Los Angeles, CA 90089-0371, USA

Full list of author information is available at the end of the article
}

for copepods, and existing models are likely to be of limited use in this group.

The overwhelming majority of arthropod model systems are insects. Additionally, Daphnia pulex (a branchiopod crustacean) has recently been sequenced [3]. However, neither insects nor Daphnia are phylogenetically close to copepods. While the precise topology of the arthropod phylogeny is currently debated, the split between Insecta, Copepoda and Branchiopoda is deep in the Cambrian. Shrimp (decapod crustaceans) are somewhat more closely related to copepods and have been the subject of genetic work [e.g. 4, 5] due to their economic importance in the seafood market. However, like other decapods, shrimp have high rates of genomic duplication, complicating genomic analyses and rendering them less useful as models [6-8].

\section{Biomed Central}


Tigriopus californicus is an increasingly attractive genetic model copepod and model crustacean. It has a history as an experimental system for environmental stress, ecotoxicology and speciation [9-12], is easily cultured in the lab, and has an experimentally tractable life cycle. It also has one of the smaller known crustacean genomes-a full order of magnitude smaller than the tiger shrimp Penaeus monodon [5].

T. californicus has an extensive range along the west coast of North America, from Alaska to Baja California. It is a supralittoral marine harpacticoid copepod, inhabiting splash pools of the rocky shore. It has extremely limited gene flow, with strong genetic differentiation between even geographically close populations $[13,14]$. Populations from Santa Cruz and San Diego, California are the focus of concerted efforts for the development of genetic resources by several labs. The transcriptomes of the two populations have recently been published [10]. A linkage map comprising 8 microsatellite markers and 45 nuclear SNPs has also recently been constructed [15] with a corrected map length of $484.8 \mathrm{cM}$, where the 11 linkage groups and one unlinked marker apparently corresponded to the 12 chromosomes of $T$. californicus [16].

A linkage map is a reference point for understanding the genetic basis of phenotypic variation. It is a valuable tool for genome-wide scans of genetic variation, for dissection of QTL (quantitative trait loci) and for the assembly of a physical map. By focusing on developing gene-based markers, our map will facilitate comparative studies using orthologous genes.

We here develop a linkage map between the Santa Cruz and San Diego populations, comprising 190 SNP markers. We validate these markers in 9 additional, geographically distinct populations of $T$. californicus. We also screen the markers in two commonly studied congeners with similar ecology: T. japonicus, which is distributed around the Asian Pacific rim, and $T$. brevicornis, which is distributed from Portugal to Nova Scotia around the Northern Atlantic. Many of the markers we develop are also reliably detectable in these two congeners, and will be broadly useful as a community resource for genetic mapping in these species. The development of this linkage map is an essential prelude to the more detailed genomic characterization of a model copepod.

\section{Methods}

\section{SNP development}

The majority of SNPs were identified from $454 \mathrm{cDNA}$ sequence data for the Santa Cruz (SC, 36 $57^{\prime} \mathrm{N}, 122^{\circ}$ $\left.03^{\prime} \mathrm{W}\right)$ and San Diego (SD, $\left.32^{\circ} 45^{\prime} \mathrm{N}, 117^{\circ} 15^{\prime} \mathrm{W}\right)$ T. californicus populations [10]. Sequences were aligned using the gsAssembler (Roche) and visualized in Consed [17].
Diagnostic SC/SD fixed SNPs were chosen using the following criteria: contig $>200 \mathrm{bp}$, minimum of 2 reads for each population, significant sequence alignment to metazoan sequence (BLAST2GO [18]) with an E-value $\leq 10^{-3}$ and sufficient conserved flanking regions for priming sites. Another subset of potential SNPs were identified from known gene sequences obtained from Genbank or from unpublished data (R. Burton). For each of the markers with a positive genetic identification, the name of the marker is consistent with the putative-orthologue name, taken as the most-significant alignment using tBlastx at [19]. The one exception is TFAM, where the most significant alignment is to a predicted gene product in Tribolium castaneum of the general class of transcription factors which includes TFAM. Subsequent best alignments are to TFAM orthologues in a number of species.

Primers and extension sequences for iPlex high throughput genotyping were developed from this dataset by Jeffrey Conroy at the Genomic Shared Resources, Roswell Park Cancer Institute, Buffalo, NY, using the iPlex Gold software (Sequenom, San Diego CA). Primer and extension sequences are shown in Additional File 1. 201 SNP assays were designed for multiplexing in a total of 7 wells, with a maximum of 30 assays in each well. Sequences ranged from 15-29bp. Within each well, the minimum molecular weight of a sequence was 4567 daltons, and the maximum weight was 8949.9 daltons (Additional File 1). The minimum difference between any two products was 16 daltons. Sequences were scored for molecular weight in a Sequenom mass spectrometer, and SNP identity determined by molecular weight.

SNPs were validated for 5 adult males and 5 adult females from each of the SC and SD lines, and found to be fixed for alternate alleles in each population, with heterozygotes confirmed in $20 \mathrm{~F}_{1}$ hybrids. SNPs were also tested in 9 additional populations of $T$. californicus (Table 1), and a single population each of T. brevicornis and $T$. japonicus (average $\mathrm{n}=5$ ). A panel of 46 previously utilized SNPs [15] was also included, including 2 mitochondrial markers to test for contamination.

\section{DNA extraction}

Nauplii (larvae) for genotyping were hatched from latestage egg sacs isolated in single drops of seawater in a petri plate. The petri plate was flooded with lysis buffer (10 mM Tris $\mathrm{pH} \mathrm{8.3,} 50 \mathrm{mM} \mathrm{KCl,} \mathrm{0.5 \%} \mathrm{Tween} \mathrm{20).}$ Individual nauplii were pipetted in $2-5 \mu \mathrm{l}$ of buffer to $200 \mu \mathrm{l}$ PCR tubes containing $10 \mu \mathrm{l}$ of buffer with 200 $\mu \mathrm{g} / \mathrm{ml}$ Proteinase $\mathrm{K}$ (final concentration). Tubes were incubated at $65^{\circ} \mathrm{C}$ for $1 \mathrm{hr}$ followed by $100^{\circ} \mathrm{C}$ for $15 \mathrm{~min}$ and then frozen at $-80^{\circ} \mathrm{C}$. DNA extraction of adult individuals followed a similar procedure except that individuals were briefly rinsed in $\operatorname{diH}_{2} \mathrm{O}$ and blotted dry on 
Table 1 Patterns of marker segregation among populations of $T$. californicus and two sister species.

\begin{tabular}{|c|c|c|c|c|c|c|c|c|c|c|c|c|c|c|c|}
\hline $\begin{array}{l}\text { pop } \\
(n=)\end{array}$ & $\begin{array}{l}\text { SD } \\
\text { (20) }\end{array}$ & $\begin{array}{l}\text { SC } \\
\text { (19) }\end{array}$ & $\begin{array}{l}\text { PA } \\
\text { (5) }\end{array}$ & & $\begin{array}{l}\text { LMC } \\
\text { (4) }\end{array}$ & $\begin{array}{l}\text { RP } \\
\text { (5) }\end{array}$ & $\begin{array}{l}\text { CAT } \\
\text { (5) }\end{array}$ & $\begin{array}{l}\text { LC } \\
\text { (5) }\end{array}$ & $\begin{array}{l}\mathrm{SCl} \\
\text { (5) }\end{array}$ & $\begin{array}{l}\text { CAR } \\
\text { (5) }\end{array}$ & $\begin{array}{l}\text { AG } \\
\text { (10) }\end{array}$ & $\begin{array}{l}\text { WIZ } \\
\text { (10) }\end{array}$ & $\begin{array}{l}\text { T.bv } \\
\text { (5) }\end{array}$ & & $\begin{array}{l}\text { T.jp } \\
\text { (5) }\end{array}$ \\
\hline amp & 190 & 190 & & 144 & 148 & 188 & 187 & 189 & 187 & 153 & 153 & 153 & & 68 & 92 \\
\hline$\overline{S D}$ & 0 & 190 & 88 & & 75 & 108 & 108 & 114 & 120 & 129 & 126 & 131 & 25 & & 48 \\
\hline SC & 12 & 0 & 33 & & 58 & 79 & 67 & 67 & 57 & 3 & 18 & 20 & 23 & & 26 \\
\hline PA & 12 & 12 & 25 & & 34 & 37 & 32 & 32 & 30 & 25 & 28 & 28 & 15 & & 20 \\
\hline$M C$ & 12 & 12 & 12 & & 15 & 17 & 17 & 19 & 22 & 46 & 46 & 46 & 22 & & 25 \\
\hline $\mathrm{RP}$ & 12 & 12 & 12 & & 12 & 2 & 13 & 17 & 27 & 52 & 49 & 51 & 23 & & 29 \\
\hline CAT & 12 & 12 & 12 & & 12 & 12 & 13 & 10 & 23 & 45 & 42 & 43 & 21 & & 27 \\
\hline LC & 12 & 12 & 12 & & 12 & 10 & 10 & 10 & 22 & 42 & 40 & 42 & 23 & & 27 \\
\hline $\mathrm{SCl}$ & 11 & 12 & 12 & & 12 & 12 & 12 & 12 & 11 & 36 & 32 & 32 & 16 & & 27 \\
\hline CAR & 12 & 12 & 12 & & 12 & 12 & 12 & 12 & 12 & 21 & 5 & 5 & 20 & & 20 \\
\hline AG & 12 & 12 & 12 & & 12 & 12 & 12 & 10 & 10 & 12 & 9 & 0 & 20 & & 23 \\
\hline WIZ & 12 & 11 & 11 & & 12 & 12 & 11 & 12 & 12 & 12 & 12 & 2 & 20 & & 23 \\
\hline T.brev & 12 & 12 & 12 & & 12 & 11 & 11 & 12 & 12 & 12 & 12 & 12 & 20 & & 5 \\
\hline T.jp & 12 & 12 & 12 & & 12 & 12 & 12 & 5 & 12 & 12 & 11 & 12 & 11 & & 18 \\
\hline
\end{tabular}

Number of fixed differences in SNPs between populations (above diagonal); number of polymorphic SNPs within population (diagonal); number of chromosomes between populations potentially distinguishable by the full panel of 190 SNP markers, including fixed differences and polymorphic alleles (below diagonal).

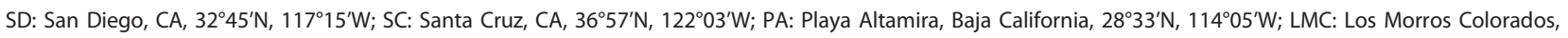

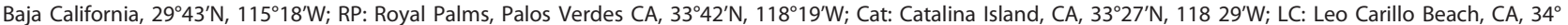

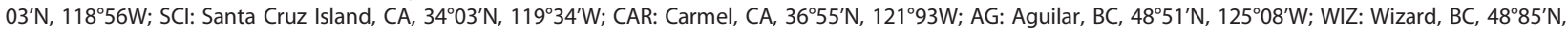
$125^{\circ} 16 \mathrm{~W}$; T.bv: Tigriopus brevicornus; T.jp: Tigriopus japonicus.

filter paper (egg sacs were gently removed from females) before being frozen at $-80^{\circ} \mathrm{C}$. Adult samples were subsequently extracted in $50 \mu \mathrm{l}$ lysis buffer with $200 \mu \mathrm{l} / \mathrm{ml}$ Proteinase $\mathrm{K}$ (final concentration). $20 \mu \mathrm{l}$ of each sample was dried at $60^{\circ} \mathrm{C}$ for 4 hours before being sent to the Roswell Park Cancer Institute for genotyping, and the remaining $30 \mu \mathrm{l}$ was retained at $-80^{\circ} \mathrm{C}$ for future work.

\section{$F_{2}$ mapping population}

Upon maturation, males of $T$. californicus perform mate guarding-holding an immature female until she is mature, then mating with her and releasing her. Observing clasped dyads facilitates sexing of immature individuals, and allows the ready collection of virgins. Females are known to mate only a single time in their life, and use stored sperm to fertilize multiple clutches of eggs [20]. Inbred-isofemale-lines are therefore established by isolating a single fertilized female and allowing offspring, including those from overlapping generations, to mate freely.

Isofemale lines were established from SC and SD populations and were maintained in the laboratory for 2.5 years. With a minimum generation time of 23 days, at $20^{\circ} \mathrm{C}$, this corresponds to approximately 39 generations of culture. All laboratory stocks were reared in a standard medium of thrice-filtered ( $40 \mu \mathrm{m}$ nitex filters) seawater with added growth medium $(0.1 \mathrm{~g}$ of spirulina and $0.1 \mathrm{~g}$ of ground Tetramin per litre of seawater). Crosses between SD females and SC males were conducted in $250 \mathrm{ml}$ beakers containing $200 \mathrm{ml}$ of growth medium. Six replicate crosses consisted each of 50 males and 50 females. Males were discarded after 1 week. Females were removed after 2 weeks, when copepodids (juveniles) became visible. When clasping pairs of $F_{1}$ offspring were observed, the males and females were separated and then united with a partner descending from a different beaker to avoid potential full sibling mating (see Additional File 2). Single pairs comprising an $F_{1}$ female and an $F_{1}$ male were placed in separate petri plates containing $12 \mathrm{ml}$ of culture medium. Males were removed when an egg sac was observed, and females moved to fresh plates as each successive clutch hatched. 250 first instar nauplii were collected for genotyping. In previous experiments, little segregation distortion has been observed in early stage nauplii [15].

\section{Additional samples for genotyping}

In addition to the $\mathrm{F}_{2}$ hybrids we also genotyped 19 nonrecombinant backcross hybrid adults [ $(\mathrm{SD}$ female $\times \mathrm{SC}$ male) $F_{1}$ female $\times S D$ male; see Additional File 2] Because T. californicus females do not undergo recombination $[16,21]$ this non-recombinant cross allows confirmation that linkage groups identified from the $F_{2}$ mapping population are on different chromosomes. Lastly, between 5 and 10 adult individuals from 9 other populations of $T$. californicus, as well as T. brevicornis and $T$. japonicus were collected for genotyping.

\section{Mapping}

The use of previously mapped SNPs [15] and cytochrome-c [22] enabled us to maintain chromosomal identity from previous work. Chromosomes 1, 2, 5, 7, 8, 
9, 10, 11 and 12 were anchored to the linkage groups of Harrison and Edmands [23] and Pritchard et al. [15] (where chromosome 12 corresponds with linkage group D). Our current chromosomes 3, 4 and 6 correspond to the linkage groups of $\mathrm{A}, \mathrm{B}$, and $\mathrm{C}$ respectively of Pritchard et al. [15] and we were not able to anchor them to Harrison and Edmands [23].

The linkage map was constructed using the JoinMap 3.0 software package [24]. The Kosambi mapping function was used, with an LOD threshold of 1.0, a recombination threshold of 0.4 , and default values for all other settings. Correct assignment of markers to chromosomes was confirmed using the non-recombinant backcross genotypes. Several markers exhibited substantial apparent segregation distortion, departing significantly from Hardy Weinberg expectation $\left(\chi^{2}>14, \mathrm{p}<0.001\right.$, df $=1$ ). We did not exclude these $a$ priori from the analysis. Following construction of the map, we examined the proposed haplotypes and found that, for the 5 most distorted markers we would have to posit recombination in females at levels above $25 \%$ between the distorted markers and their nearest neighbors to explain the marker states. As recombination in females is known to not occur at levels $>1 \%[16,21]$, we excluded these 5 markers from the analysis. We were unable to determine the source of this apparent genotyping error, but one possibility is cryptic, previously unidentified polymorphism.

\section{Predicted effect of SNPS on protein sequence}

Nucleotides that are under lower selective constraint might be expected to have higher substitution rates, and thus may be expected to have more alleles across populations. For all SNPs, we used the probable coding sequences of Barreto et al. [10] to determine whether the SNP was in a translated region of the transcript, and if so, whether or not the SNPs were synonymous. SNP positions and translations were calculated by matching the Sequenom extension sequences against the transcripts of Baretto et al [10] using the Bioperl (v1.6.1) module of Perl (v 5.12.2).

\section{Synteny with model species}

We attempted to find signs of macrosynteny with several assembled arthropod genomes-the insects Apis mellifera [25], assembly 4.5, with a $1 \mathrm{n}=16$; D. melanogaster [26] assembly 4.3, with $1 \mathrm{n}=5$ (commonly the major chromosomal arms are treated as independent linkage groups); and Tribolium castaneum [27] assembly 3.0 with a $1 n=12$; and the recently sequenced Branchiopod crustacean, Daphnia pulex [3] beta 3 release with a $1 \mathrm{n}=12$. Of these, the insect genomes were the most fully assembled, and we were able to disregard any unanchored scaffolds. In $D$. pulex, there were far more unanchored scaffolds-3804-and we did not exclude any (although the majority of the genome is assembled in the first 50 scaffolds and the majority of our Blast alignments were expected to localize on these).

To identify homologs, we performed sequence alignment searches using tBlastx of the flanking sequence for each of our mapped markers (cDNA contigs for new markers and ESTs for markers from Pritchard et al. [15]) against each reference draft genome assembly, with a threshold of E-value $\leq 10^{-3}$ and the default settings. Several sequences had multiple alignments exceeding this threshold, and in these cases we considered only the most significant sequence alignment. The $A$. mellifera amino acid sequences were obtained from[28]. The $T$. castaneum amino acid sequences were obtained from [29], and the chromosomal location of the most significant alignments for both the $A$. mellifera and $T$. castaneum genomes were obtained by querying the Batch Entrez database at [19] with the gi numbers. The D. melanogaster v. 4.3 sequences were obtained from Flybase [30]. The D. pulex amino acid sequences were obtained from [31] and the scaffold identity of these sequences subsequently obtained by tBlastn to the genomic scaffold obtained at the same site. Where a pair of putative homologs colocalized to a single linkage group in both species, we scored it as a single potential syntenic unit. Where three markers colocalized in this way, we scored 2 alignments (in no case did more than 3 homologs colocalize in both species). We summed all potential syntenic units for each species (Table 2). Significance was determined by randomly permuting the homolog locations relative to the $T$. californicus chromosomal map positions 1000 times in R [32], and counting the number of estimated syntenic units each time.

\section{Results}

\section{SNP development}

Of the total of 245 nuclear SNPs we assayed, 190 SNPs were found to be reliable in our genotyping pools, including 38 of the previously developed markers [15]. Only these 190 SNP markers are reported here (Additional File 1). In nauplii, none of these markers were found to be significantly distorted after Bonferroni correction. Primers and extension sequences were found to be widely conserved within populations of T. californicus, as well as sister species T. brevicornis and T. japonicus. Many of the SNPs identified in the SC-SD comparison were polymorphic within other populations (Table 1, diagonal, bold; Additional File 1). The number of fixed differences between populations, as well as segregating polymorphisms within populations, together represent the potential pool of markers for mapping in T. californicus. The number of potential markers for use in mapping differences between population pairs appears in the above-diagonal of Table 1. 
Table 2 Number of pairs of colocalizing loci in both Tigriopus californicus and assembled reference genomes.

\begin{tabular}{lrrrrr}
\hline & linkage groups & $\mathbf{n}$ align & $\mathbf{n}$ pairs & threshold & $\mathbf{p}$-value range \\
\hline Apis mellifera & 16 & 124 & 46 & 43 & $\mathbf{0 . 0 0 7 - 0 . 0 0 2}$ \\
Tribolium castaneum & 10 & 134 & 55 & 61 & $0.649-0.535$ \\
Drosophila melanogaster & 5 & 144 & 91 & 94 & $0.563-0.369$ \\
Daphnia pulex & $>3000$ & 72 & 2 & 4 & $0.607-0.321$ \\
\hline
\end{tabular}

The number of linkage groups of the draft reference genomes of each species are shown, with the number of identified homologues between $T$. californicus and each of the reference genomes, and the number of homologue pairs which colocalize to a linkage group in both $T$. californicus and the reference. The maximum (threshold) number of colocalized pairs we would expect to see by chance, and the significance of the observed number of colocalized pairs were determined by permutation.

\section{Mapping}

The final assembly consisted of 12 linkage groups, corresponding to the known 12 chromosomes of $T$. californicus (Figure 1). These 12 linkage groups were confirmed by results from the non-recombinant backcross. The average intermarker distance was $1.81 \mathrm{cM}$, and the maximum distance between adjacent markers was $10.3 \mathrm{cM}$. The sum of the distances between the markers gives an estimated linkage map length of $323.5 \mathrm{cM}$ long. Applying the suggested correction method [number 4 in 33] to estimate total map length, where $L_{i}$ is map length of a given chromosome $i$, and $\mathrm{m}_{\mathrm{i}}$ is the number of markers for that linkage group,
$L_{\text {tot }}=\sum_{i}\left(L_{i+2}\right) \times(m+1) /(m-1)=401.0 \mathrm{cM}$. We estimated total coverage following [34]. Given total marker number $\mathrm{n}=190$, and average intermarker distance $\mathrm{d}=1.81 \mathrm{cM}$, the proportion coverage $c=1-e^{-2 d n / L_{\text {tot }}}=0.737$. The recombination rate, given the estimated haploid genome size of $244.5 \mathrm{Mb}[35,36]$, corresponds approximately $1.6 \mathrm{cM} / \mathrm{Mbp}$. The sizes of the linkage groups ranged from 19 to $32 \mathrm{cM}$.

\section{Predicted effect of SNPs on protein sequence}

Of the 190 SNPs used to construct the linkage map, 125 (66\%) are predicted to be translated, and of these 125 , $101(79.5 \%)$ are synonymous substitutions. Of these 101

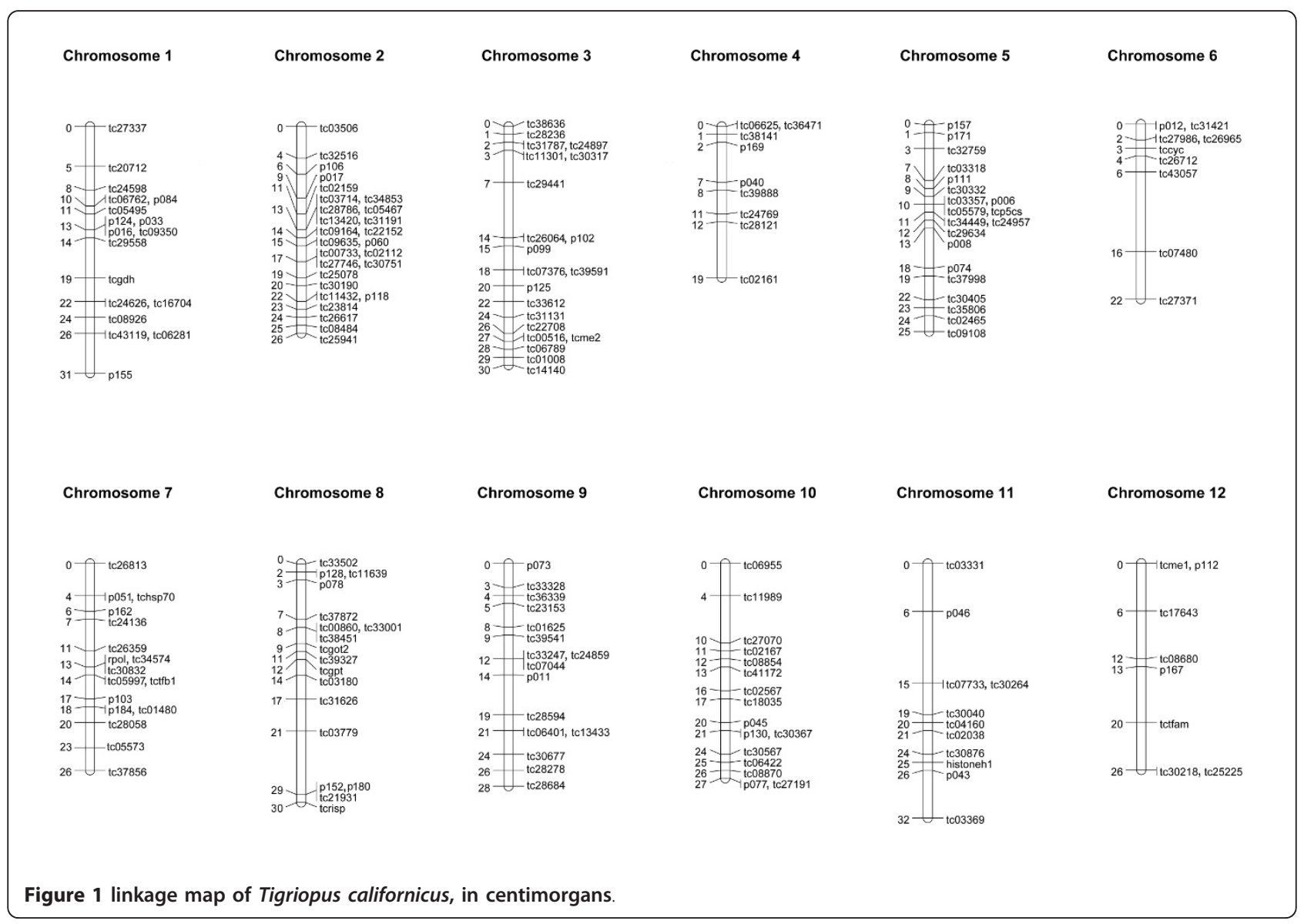


loci, there are other synonymous substitutions possible at 80 loci (Additional File 1).

\section{Synteny with model species}

Orthologues to 153 of the 190 T. californicus sequences were detected in the reference genomes (Additional File 3 ). The greatest number of detected orthologues (144) was with reference to the $D$. melanogaster genome, and the lowest (72) was with $D$. pulex. There was evidence of macrosynteny between T. californicus and the A. mellifera genome. Nine pairs of orthologous genes were found to colocalize on chromosomes in both species, above the maximum of 7 pairs we might expect to find colocalizing by chance. After applying a Bonferroni correction for 4 tests, for each of the reference genomes, the confidence interval for the number of syntenic units shared between the two species is still below the $\mathrm{p}=$ 0.0125 threshold for significance as determined by permutation (Table 2, Table 3).

\section{Discussion}

We successfully mapped the T. californicus nuclear genome, using 38 previously described SNPs, and 152 novel SNPs, to a total of 190 markers. This corresponds to an average of 1 marker every $1.29 \mathrm{Mbp}$, and an average intermarker distance of $1.8 \mathrm{cM}$. All markers were assigned to linkage groups, and the maximum intermarker distance was $10.3 \mathrm{cM}$. We will thus be able to use these markers to impute genotypes in interval mapping with $95 \%$ accuracy or better for all positions in the genome. The 12 linkage groups obtained corresponded to the 12 known chromosomes of T. californicus, and most were anchored to linkage groups previously identified with microsatellite and Amplified Fragment Length Polymorphism (AFLP) markers [23]. The total corrected map length of $401.0 \mathrm{cM}$ was somewhat lower than the estimated map length of Pritchard et al. [15], and the estimated total coverage of $73.7 \%$ was also somewhat lower than their $75.2 \%$ coverage, but this difference in

Table 3 Chromosomes with colocalizing homologous sequences in $T$. californicus and $A$. mellifera.

\begin{tabular}{crrrr}
\hline T. californicus chromosome & \multicolumn{4}{c}{ A. mellifera chromosomes } \\
\hline 1 & $1(3)$ & $14(2)$ & - & - \\
2 & $4(2)$ & $11(2)$ & $12(2)$ & $15(3)$ \\
3 & $1(2)$ & $6(3)$ & $11(2)$ & - \\
4 & $15(2)$ & - & - & - \\
5 & $1(4)$ & $2(3)$ & $15(2)$ & - \\
6 & $8(3)$ & - & - & - \\
7 & $1(2)$ & $10(3)$ & $11(3)$ & - \\
8 & $1(4)$ & $6(2)$ & $8(3)$ & $10(2)$ \\
9 & $7(2)$ & $11(3)$ & $16(2)$ & - \\
10 & $1(3)$ & $3(2)$ & $4(2)$ & $5(3)$ \\
\hline
\end{tabular}

estimates is unsurprising given the much deeper coverage we have achieved in the current study.

All crosses between populations of $T$. californicus from Southern California to British Columbia produce fertile $F_{1}$ progeny [37]. Pairs of populations, however, exhibit increasing $\mathrm{F}_{2}$ hybrid breakdown by distancepopulations as close as $100 \mathrm{~km}$ can have a reduction in fitness of $50 \%$ in the $\mathrm{F}_{2}$ relative to the parental populations $[13,37]$. We found no evidence of segregation distortion in the nauplii of our mapping population, however. This is consistent with previous studies which found generally undistorted allele frequencies in the early larval stage, while adults had allele frequencies which strongly departed from expected frequencies $[15,38]$.

Primer sequences used for polymerase chain reaction DNA amplifications were largely conserved between populations. In only one population (PA) did more than 5 primer sets fail to amplify a product, and we found many SNPs segregating within and between populations. The elevated differentiation between PA and the Southern Californian populations was not unexpected, as Baja populations of $T$. californicus have been found to be very divergent in mitochondrial haplotype [13], and to exhibit high rates of hybrid breakdown when crossed with northern populations [39]. Many of our primers also amplified products in the sister species $T$. japonicus and T. brevicornis. The SNP markers we have characterized here, therefore will be generally useful for QTL mapping across the entire geographic range of $T$. californicus. For all but a few population pairs, it will be possible to construct mapping lines and distinguish every chromosome in a non-recombinant backcross design.

Additional SNPs may be found to segregate in these populations, since they were only genotyped for the known SD and SC SNPs (or SC, SD and a third population in the case of the SNPs from Pritchard et al. [15]). Only 20 of the SNP markers were in coding regions at twofold degenerate sites, suggesting as many as $89.5 \%$ of the SNPs may be under relaxed selective constraint. Populations will thus need to be characterized for alternate alleles for mapping purposes. The known level of triallely of SNPs is very low in T. californicus, however. V. Pritchard (pers. comm) found that between San Diego, Santa Cruz and Punta Baja (Baja California), levels of triallely are approximately $2 \%$ at SNP sites across the genome. These 3 populations are deeply divergent within $T$. californicus, suggesting that withinspecies at least, there will be few uncharacterized alleles.

Compared with the other arthropods, the recombination rate of $T$. californicus (1.6 cM/Mbp) is low [reviewed in 39], but is more than sufficient for mapping in $F_{2}$, backcross and other linkage mapping approaches. It is similar to that of Diptera $($ mean $=1.03$ 
$\mathrm{cM} / \mathrm{Mbp}$ ) and shrimp Penaeus monodon (mean $=1.01$ cM/Mbp)[5], but it is approximately $21 \%$ the estimated recombination rate of Daphnia pulex at $7.52 \mathrm{cM} / \mathrm{Mbp}$ [40]. It is much lower than Hymenoptera (mean $=7.12$ $\mathrm{cM} / \mathrm{Mbp}$ ), and somewhat lower than the more typical arthropod recombination rate of Coleoptera (mean = $2.48 \mathrm{cM} / \mathrm{Mbp}$ ) [41]. It is known that recombination rates in groups with alternate life histories are under selection-for instance, social vs. solitary Hymenoptera have greatly elevated recombination rates. The relatively high recombination rate of $D$. pulex compared to shrimp and T. californicus is possibly due to its reproductive mode-many generations of parthenogenesis, punctuated by episodic sex, may have consequences on the evolution of recombination rate [42]. Another reason for the relatively low recombination rate might be that recombination occurs only in males in T. californicus $[16,21]$.

Additionally, perhaps surprisingly, there was some evidence of macrosynteny between A. mellifera and T. californicus, although there was no one-to-one correspondence between chromosomes. No evidence of synteny was found T. californicus and D. pulex or $D$. melanogaster; however, this may be because these genomes have undergone higher rates of chromosomal rearrangements. It was reported that no evidence of synteny was found between $D$. pulex and D. melanogaster either [40]. In fact, Critescu et al. [40] suggest-based on the rearrangement rate of Drosophila-that synteny should only be apparent between crustaceans and insects at scales smaller than $100 \mathrm{~kb}$. It may be that Hymenoptera and Copepoda have lower rates of large-scale chromosomal fission and fusion than Drosophila and the other assayed arthropod lineages.

\section{Conclusion}

We have mapped the T. californicus genome to a level of coverage that will enable common mapping techniques such as nonrecombinant backcross, and $F_{2}$ or Recombinant Inbred Line mapping-greatly expanding the possible scope of work in this species. The markers and map developed will potentially be useful more broadly in the genus Tigriopus. The phylogenetic position of T. californicus, its compact genome size, and its tractability in the lab have all positioned it as an excellent model crustacean system. The continued development of genomic tools such as this linkage map will enable future exciting work in this species.

\section{Abbreviations used}

A. mellifera: Apis mellifera; D. pulex: Daphnia pulex; $P$. monodon: Penaeus monodon; T. castaneum: Tribolium castaneum; QTL: Quantitative Trait Locus; SNP: Single Nucleotide Polymorphism; T. californicus/japonicus/ brevicornis: Tigriopus spp.; cDNA: coding DNA. Populations: AG: Aguilar, BC, $48^{\circ} 51^{\prime} \mathrm{N}, 125^{\circ} 08^{\prime} \mathrm{W}$; CAR: Carmel, CA, $36^{\circ} 55^{\prime} \mathrm{N}, 121^{\circ} 93 \mathrm{~W}$; Cat: Catalina Island, CA, $33^{\circ} 27^{\prime} \mathrm{N}, 118$ 29’W; LC: Leo Carillo Beach, CA, 3403’N, 118 56W; LMC: Los Morros Colorados, Baja California, $29^{\circ} 43^{\prime} \mathrm{N}, 115^{\circ} 18^{\prime} \mathrm{W}$; PA: Playa Altamira, Baja California, $31^{\circ} 52^{\prime} \mathrm{N}, 116^{\circ} 40^{\prime} \mathrm{W}$; RP: Royal Palms, Palos Verdes CA, $33^{\circ} 42^{\prime} \mathrm{N}, 118^{\circ} 19^{\prime} \mathrm{W}$; SC: Santa Cruz, CA $36^{\circ} 57^{\prime} \mathrm{N}, 122^{\circ}$ $03^{\prime} \mathrm{W}$; SD: San Diego, CA, $32^{\circ} 45^{\prime} \mathrm{N}, 117^{\circ} 15^{\prime} \mathrm{W}$; SCI: Santa Cruz Island, CA, $34^{\circ} 03^{\prime} \mathrm{N}, 119^{\circ} 34^{\prime} \mathrm{W}$; WIZ: Wizard, BC, $48^{\circ} 85^{\prime} \mathrm{N}, 125^{\circ} 16 \mathrm{~W}$.

\section{Additional material}

Additional File 1: "Table of markers used to create the linkage map.
Showing calculated map positions, forward and reverse primer
sequences, extension sequences with associated SD and SC alleles.
Marker genotypes of a wide geographic sampling of populations, as well
as sister species, are shown. Also shown is the sequence description
from the most significant alignment obtained by Blast search (GenBank).
Inferred San Diego and Santa Cruz triplet codons are shown, with the
SNP position and amino acid translation. If SNPs are predicted to be
synonymous, the number of additional synonymous alleles at the SNP
site is indicated."
Additional File 2: "Crossing scheme between San Diego (SD) and
Santa Cruz (SC) to generate F2 and non-recombinant backcross
(nrBC) mapping lines in Tigriopus californicus."
Additional File 3: "Table of most significant tBlastx alignments (E-
value $\leq 10$-3) between T. californicus sequences and the reference
draft genome assemblies of model species Apis mellifera, Tribolium
castaneum, Drosophila melanogaster and Daphnia pulex. For each
significant alignment are shown the gi number, the E-value of the
alignment, and the linkage information of the homologue.

Acknowledgements and funding

This work was funded by U.S. National Science Foundation grants DEB0743472 to SE and DEB-0717178 to RSB, and by a USC Women in Science and Engineering grant to SE. Many thanks to Jeffrey Conroy for his help with the SNP assays and to Grace Jang and Tara Matsuda for laboratory assistance.

\section{Author details}

${ }^{1}$ Department of Biological Sciences, University of Southern California, Los Angeles, CA 90089-0371, USA. Windward School, Los Angeles, CA 900662104, USA. ${ }^{3}$ Department of Organismic and Evolutionary Biology, Harvard University, Cambridge, MA 01730, USA. ${ }^{4}$ Marine Biology Research Division, Scripps Institution of Oceanography, University of California, San Diego, La Jolla, CA 92037, USA.

\section{Authors' contributions}

SE designed the study, assisted with crosses, oversaw analyses and contributed to the final written manuscript. GWM and RSB generated transcriptome data. CGR developed the SNP assays, constructed the linkage map and assisted with crosses. DER and WL assisted with crosses. BRF performed all post-map analyses and wrote the manuscript. All authors contributed comments and approved the manuscript prior to submission.

Received: 24 May 2011 Accepted: 21 November 2011

Published: 21 November 2011

\section{References}

1. Humes AG: How many copepods? Hydrobiologia 1994, 292-293:1-7.

2. Turner JT: Pelagic Marine Food Webs. Zoological Studies 2004, 43:255-266. 
3. Colbourne JK, Pfrender ME, Gilbert D, Thomas WK, Tucker A, Oakley TH, Tokishita S, Aerts A, Arnold GJ, Basu MK, Bauer DJ, Caceres CE, Carmel L, Casola C, Choi J-H, Dong Q, Dusheyko S, Eads BD, Frohlich T, GeilerSamerotte KA, Gerlach D, Hatcher P, Jogdeo S, Krijgsveld J, Kriventseva EV, Kultz D, Laforsch C, Lindquist E, Lopez J, et al: The Ecoresponsive Genome of Daphnia pulex. Science 2011, 331:555-561.

4. Du ZQ, Ciobanu DC, Onteru SK, Gorbach D, Mileham AJ, Jaramillo G, Rothschild MF: A gene-based SNP linkage map for pacific white shrimp, Litopenaeus vannamei. Animal genetics 2010, 41:286-94.

5. You EM, Liu KF, Huang SW, Chen M, Groumellec ML, Yu HT: Construction of integrated genetic linkage maps of the tiger shrimp (Penaeus monodon) using microsatellite and AFLP markers. Animal genetics 2010, 41:365-76.

6. Otto SP, Whitton J: Polyploid incidence and evolution. Annual Review of Genetics 2000, 34:401-437.

7. Koyama T, Asakawa S, Katagiri T, Shimizu A, Fagutao FF, Mavichak R, Santos MD, Fuji K, Sakamoto T, Kitakado T, Kondo H, Shimizu N, Aoki T, Hirono I: Hyper-expansion of large DNA segments in the genome of kuruma shrimp, Marsupenaeus japonicus. BMC genomics 2010, 11:141.

8. Lecher P, Defaye D, Noel P: Chromosomes and nuclear DNA of Crustacea. Invertebrate Reproduction \& Development 1995, 27:85-114

9. Raisuddin S, Kwok KWH, Leung KMY, Schlenk D, Lee J-S: The copepod Tigriopus: a promising marine model organism for ecotoxicology and environmental genomics. Aquatic toxicology (Amsterdam, Netherlands) 2007, 83:161-73.

10. Barreto FS, Moy GW, Burton RS: Interpopulation patterns of divergence and selection across the transcriptome of the copepod Tigriopus californicus. Molecular ecology 2011, 20:560-72.

11. Burton RS, Metz EC, Flowers JM, Willett CS: Unusual structure of ribosomal DNA in the copepod Tigriopus californicus: intergenic spacer sequences lack internal subrepeats. Gene 2005, 344:105-13.

12. Willett CS: Potential fitness trade-offs for thermal tolerance in the intertidal copepod Tigriopus californicus. Evolution 2010, 2521-2534

13. Edmands S: Phylogeography of the intertidal copepod Tigriopus californicus reveals substantially reduced population differentiation at northern latitudes. Molecular ecology 2001, 10:1743-50

14. Burton RS, Feldman MW, Curtsinger JW: Population genetics of Tigriopus californicus (Copepoda: Harpacticoida): I. Population structure along the central California coast. Marine Ecology Progress Series 1979, 1:29-39.

15. Pritchard VL, Dimond L, Harrison JS, Velázquez CCS, Zieba JT, Burton RS, Edmands S: Interpopulation hybridization results in widespread viability selection across the genome in Tigriopus californicus. BMC Genetics 2011, 12:54.

16. Ar-Rushdi AH: The cytology of achiasmatic meiosis in the female Tigriopus (Copepoda). Chromosoma 1963, 13:526.

17. Gordon D, Abajian C, Green P: Consed: a graphical tool for sequence finishing. Genome Research 1998, 8:195-202

18. García-Gómez JM, Terol J, Williams TD, Nagaraj SH, Nueda MJ, Robles M, Talón M, Dopazo J, Conesa A: High-throughput functional annotation and data mining with the Blast2GO suite. Nucleic Acids Res 2008, 36(10):3420-3435.

19. The National Center for Biotechnology Information website. [http://ncbi. nih.gov].

20. Burton RS: Mating system of the intertidal copepod Tigriopus californicus. Marine Biology 1985, 86:247-252.

21. Burton RS, Feldman M, Swisher S: Linkage relationships among five enzyme-coding gene loci in the copepod Tigriopus californicus: a genetic confirmation of achiasmiatic meiosis. Biochemical genetics 1981, 19:1237-45

22. Edmands S, Northrup SL, Hwang AS: Maladapted gene complexes within populations of the intertidal copepod Tigriopus californicus? Evolution 2009, 63:2184-92.

23. Harrison JS, Edmands S: Chromosomal basis of viability differences in Tigriopus californicus interpopulation hybrids. Journal of evolutionary biology 2006, 19:2040-51.

24. van Ooijen J, Voorrips R: JoinMap ${ }^{\circledR}$ 3.0, Software for the calculation of genetic linkage maps. Plant Research International 2001.

25. Weinstock GM, Robinson GE, Gibbs RA, Worley KC, Evans JD, Maleszka R, Robertson HM, Weaver DB, Beye M, Bork P, Elsik CG, Hartfelder K, Hunt GJ, Zdobnov EM, Amdam GV, Bitondi MMG, Collins AM, Cristino AS, Michael H, Lattorff G, Lobo CH, Moritz RFA, Nunes FMF, Page RE, Simes ZLP,
Wheeler D, Carninci P, Fukuda S, Hayashizaki Y, Kai C, Kawai J, et al: Insights into social insects from the genome of the honeybee Apis mellifera. Nature 2006, 443:931-949.

26. Adams MD: The Genome Sequence of Drosophila melanogaster. Science 2000, 287:2185-2195.

27. Richards S, Gibbs RA, Weinstock GM, Brown SJ, Denell R, Beeman RW Gibbs R, Bucher G, Friedrich M, Grimmelikhuijzen CJP, Klingler M, Lorenzen M, Roth S, Schröder R, Tautz D, Zdobnov EM, Muzny D, Attaway T, Bell S, Buhay CJ, Chandrabose MN, Chavez D, ClerkBlankenburg KP, Cree A, Dao M, Davis C, Chacko J, Dinh H, Dugan-Rocha S, Fowler $\mathrm{G}$, et al: The genome of the model beetle and pest Tribolium castaneum. Nature 2008, 452:949-55.

28. The NCBI FTP site for Apis mellifera amino acid sequences. [ftp://ftp.ncbi. nih.gov/genomes/Apis_mellifera/protein].

29. Brown SJ, Caragea D: KSU Bioinformatics Center.[ftp://bioinformatics.ksu. edu/pub/BeetleBase/3.0/Sequences/Manually_Curated_CDS_Peptide_mRNA/ Manually_Curated_Peptide].

30. Flybase Genomes FTP site. [ftp://ftp.flybase.net/genomes/aaa/dmel_r4.3].

31. Indiana University Bio-Archive. [ftp://iubio.bio.indiana.edu/daphnia/ genome/Daphnia_pulex].

32. R Development Core Team: R: A language and environment for statistical computing. 2010.

33. Chakravarti A, Lasher LK, Reefer JE: A maximum likelihood method for estimating genome length using genetic linkage data. Genetics 1991, 128:175-82.

34. Turelli M, Orr HA: Dominance, epistasis and the genetics of postzygotic isolation. Genetics 2000, 154:1663-79.

35. Wyngaard GA, Rasch EM: Patterns of genome size in the copepoda. Hydrobiologia 2000, 417:43-56.

36. Gregory TR, Nicol JA, Tamm H, Kullman B, Kullman K, Leitch IJ, Murray BG, Kapraun DF, Greilhuber J, Bennett MD: Eukaryotic genome size databases. Nucleic acids research 2007, 35:D332-8[http://www.genomesize.com].

37. Edmands S: Heterosis and outbreeding depression in interpopulation crosses spanning a wide range of divergence. Evolution 1999, 53:1757-1768.

38. Willett CS, Berkowitz JN: Viability effects and not meoitic drive cause dramatic departures from Mendelian inheritance for malic enzyme in hybrids of Tigriopus californicus populations. Journal of evolutionary biology 2007, 20:1196-205.

39. Ganz HH, Burton RS: Genetic differentiation and reproductive incompatibility among Baja California populations of the copepod Tigriopus californicus. Marine Biology 1995, 123:821-827.

40. Cristescu MEA, Colbourne JK, Radivojac J, Lynch M: A microsatellite-based genetic linkage map of the waterflea, Daphnia pulex: On the prospect of crustacean genomics. Genomics 2006, 88:415-30.

41. Wilfert L, Gadau J, Schmid-Hempel P: Variation in genomic recombination rates among animal taxa and the case of social insects. Heredity 2007 98:189-97

42. Lande R: The genetic correlation between characters maintained by selection, linkage and inbreeding. Genetical research 1984, 44:309-320.

\section{doi:10.1186/1471-2164-12-568}

Cite this article as: Foley et al.: A gene-based SNP resource and linkage map for the copepod Tigriopus californicus. BMC Genomics 2011 12:568.

\section{Submit your next manuscript to BioMed Central and take full advantage of:}

- Convenient online submission

- Thorough peer review

- No space constraints or color figure charges

- Immediate publication on acceptance

- Inclusion in PubMed, CAS, Scopus and Google Scholar

- Research which is freely available for redistribution 